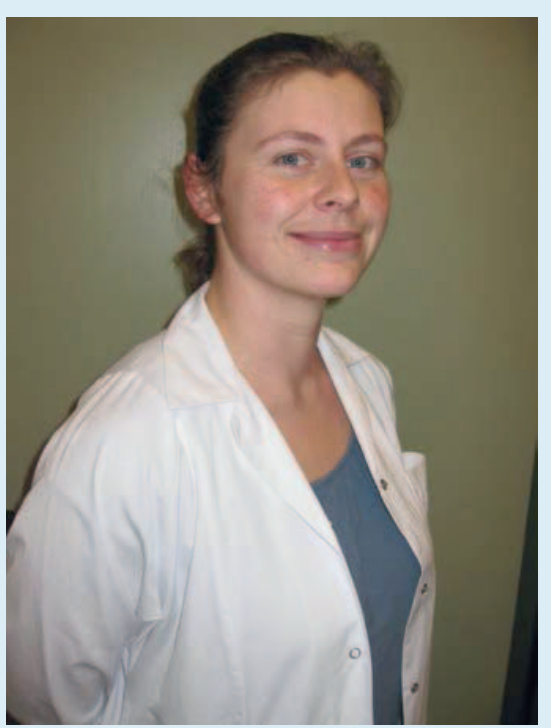

Die Zukunft

Die junge Medizinergeneration, die jetzt im Studium steckt oder gerade mit der Ausbildung fertig ist, wird in der Zukunft das Gesicht der Hausarztmedizin in der Schweiz prägen. Sie wird bestimmen, wie Patienten behandelt werden und welchen Stellenwert etwa die Ganzheitsmedizin haben wird. Einen Einblick, wie die junge Generation sich, die Medizin und die Herausforderungen von morgen und übermorgen sieht, gibt das Interview mit der Ärztin Gabriela Rohrer (Jahrgang 1983).

\title{
Wer heilt, hat recht
}

Gabriela Rohrer, Vorstandsmitglied «Junge Hausärzte Schweiz» (JHaS), schildert, wie sie als künftige Hausärztin die Zukunft sieht. Die nächste Generation der Hausärzte betrachtet sich als Dienstleister für ihre Patienten und als Schaltzentrale rund um deren Gesundheit. Der Trend, als Hausarzt im Team und in Gemeinschaftspraxen zu arbeiten, wird zunehmen. Die alte Konfrontation zwischen Schul- und Komplementärmedizin ist endgültig von gestern.

Wie viele andere Ärzte Ihrer Generation sind Sie noch in der Ausbildung. Was sind Ihre Erwartungen an Ihre Arbeit und die Organisation Ihrer Praxis, wenn Sie sich als Hausärztin niedergelassen haben?

Gabriela Rohrer: Meine Vorstellung ist sicher nicht repräsentativ in allen Punkten. Ich stelle mir vor, dass ich in einer Praxis auf dem Land arbeiten werde, in der auch die Notfall- und Rettungsmedizin eine wichtige Rolle spielt. Ich will die guten diagnostischen Möglichkeiten zur Verfügung haben, an die ich mich während meiner Ausbildung in guten Hausarztpraxen und im Spital gewöhnt habe. Auch in $\mathrm{Zu}-$ kunft braucht der Hausarzt radiologische Möglichkeiten und den $\mathrm{Zu}$ gang zu einem Labor.

Ich nehme an, dass man in $\mathrm{Zu}-$ kunft verstärkt in Teams in Gemeinschaftspraxen arbeiten wird. Vielleicht wird das Angebot der Hausarztpraxen häufiger durch die Nachbarschaft oder eine Kooperation, z.B. mit Physiotherapeuten, ergänzt. Meine ganz persönliche Vorstellung ist, dass man in einer ärztlichen Gemeinschaft dort auf dem Lande ein umfassender Grundversorger für die Bevölkerung ist, der gute und pragmatische Medizin anbietet.
Der Hausarzt als Einzelkämpfer dürfte damit der Vergangenheit angehören?

Den Hausarzt als Einzelkämpfer habe ich während meiner Ausbildung bisher gar nicht erlebt. Ich habe eine Zeit lang in Lauterbrunnen in einer Hausarztpraxis gearbeitet. Der Chef dort hatte immer einen Assistenzarzt als Praxisassistenten angestellt. Zusammen mit den Arzthelferinnen in der Praxis und dem angegliederten Physiotherapeuten hat sich so ein grosses Team mit viel Energie um die Patienten in dieser Region gekümmert Viele der Patienten leben dort im Berner Oberland auf abgelegenen Höfen. Ihre ärztliche Versorgung wäre ohne eine enge Zusammenarbeit mit der Spitex gar nicht möglich. Auch wenn viele bei der $\mathrm{Zu}-$ kunftsplanung an die Städte denken, ist ein grosser Teil der Schweiz nach wie vor ländlich. Dort braucht es andere Antworten auf die medizinischen Herausforderungen. Die Gesundheitsversorgung fernab vom Spital wird auch in Zukunft in den regional gewachsenen grösseren oder kleineren Netzwerken geleistet, wie das heute schon geschieht. Wenn die Politik neuerdings eine gute Zusammenarbeit unter den Ärzten und mit den anderen Akteuren im Gesundheitswesen fordert, verkennt sie die Realität. Ich habe den Eindruck, dass die Hausärzte in der Schweiz bereits heute sehr gut vernetzt sind und

\section{KARGER}

Fax +4976145207 14 
interdisziplinär arbeiten. Man kann davon ausgehen, dass die künftigen Hausärzte meiner Generation das fortführen werden.

Wird die Bedeutung des Hausarztes als "Schaltzentrale» für die Gesundheitsversorgung der Bevölkerung zunehmen?

Auch in Zukunft soll die Hausarztpraxis der Dreh- und Angelpunkt für die Gesundheit und die Versorgung der Bevölkerung bleiben. Entscheidend ist, dass sich genügend Ärztinnen und Ärzte finden, die bereit sind, diesen Berufsweg auf sich zu nehmen. Zurzeit fehlt vielerorts ein Praxisnachfolger. Die Praxen werden dann oftmals von Versicherungen übernommen. Die in diesen Gesundheitszentren angestellten Ärzte ziehen häufig nach relativ kurzer Zeit weiter. Eine langfristige Arzt-PatientBeziehung lässt sich weniger gut aufbauen. Zudem stimmt es mich nachdenklich, wenn der Versicherer der Brotgeber des Arztes ist. Da kommt es rasch zum Interessenkonflikt. Es ist nicht die Aufgabe einer Versicherung, die ärztliche Versorgung zu organisieren.

Wie gross schätzen Sie das Interesse der jungen Ärzte ein, sich als Hausarzt in einer Gemeinschaftspraxis niederzulassen?

Das ist schwierig zu sagen, denn in meiner Generation, die zwischen Ende zwanzig und Anfang dreissig ist, haben viele noch keine allzu konkreten Pläne für die Zeit nach der Ausbildung. Viele können sich vorstellen, später einmal in die Praxis $\mathrm{zu}$ gehen. Ich glaube, dass wir uns in dieser Phase unseres Lebens und unserer Ausbildung auch etwas treiben lassen müssen, um den Weg zu finden, den wir gehen wollen. Das haben die Generationen vor uns ja auch getan.
Heute spielt das Thema «Work-LifeBalance" eine wichtige Rolle, und die Arbeitsbelastung eines Hausarztes scheint mit den Ansprüchen an das eigene Leben nicht mehr vereinbar. Macht das den Beruf des Hauszarztes eher unattraktiv?

Die langen Arbeitszeiten machen den Arztberuf insgesamt weniger attraktiv - das gilt für die Arbeit im Spital genauso wie für die Arbeit in der eigenen Praxis. Weil sich die Lebensumstände geändert haben, braucht es neue Organisationsformen, die ihnen Rechnung tragen. Für viele Ärztinnen und Ärzte in meinem Alter, die jetzt eine Familie gründen und kleine Kinder haben, ist es eine Herausforderung, die privaten Verpflichtungen und beruflichen Interessen zu vereinbaren. In den Spitälern beginnt man zu reagieren - allerdings sehr langsam und oft mit Widerwillen. So werden Teilzeitstellen geschaffen und Job-Sharing wird ermöglicht. Die Umsetzung ist leider häufig nicht durchdacht, weil sie sich nicht am Zeitbedarf der Betroffenen orientiert. Was nützt es einer jungen

\section{Junge Hausärztinnen und -ärzte Schweiz}

Die JHaS (www.jhas.ch) ist die Organisation der angehenden und jungen Hausärztinnen und Hausärzte, die sich für die Vernetzung und die Anliegen der Ärztinnen und Ärzte in der Aus- und Weiterbildung zur Hausarztmedizin engagiert. Sie sieht sich als Interessenvertreterin der jungen medizinischen Generation der Schweiz.

Zielgruppe sind alle Medizinerinnen und Mediziner in der Aus- und Weiterbildung, die an der Hausarztmedizin Interesse haben. Gemeinsam entwickelt die JHaS Zukunftsvisionen und bringt die Anliegen der zukünftigen Hausärztegeneration in den politischen Prozess der Schweiz ein.

Gabriela Rohrer ist Mitglied im Vorstand der JHaS und dort für das Ressort Weiterbildung verantwortlich. Sie hat 2008 das Medizinstudium in Bern begonnen und ist seit September 2012 Assistenzärztin am Kantonsspital St. Gallen.
Ärztin mit Kindern, wenn sie in einer Woche $100 \%$ arbeiten muss und dafür die nächste Woche komplett frei hat? Mit solch einer Verteilung der Arbeitszeit lässt sich keine Kinderbetreuung organisieren. Auch wenn oft das Gegenteil behauptet wird: Die Arbeitsbedingungen, die im Gesundheitswesen der Schweiz herrschen, und das Familienleben in Einklang zu bringen, ist schwierig!

Hier bieten Hausarztpraxen viel bessere Möglichkeiten. Bei den niedergelassenen Hausärzten sehe ich oft einen innovativen Geist, den ich bei vielen Chefärzten in den Spitälern vermisse. Junge Ärztinnen mit Kindern, aber auch Ärzte, die ihre Verantwortung als Väter wahrnehmen wollen, finden hier gute Möglichkeiten, nur an bestimmten Tagen oder nur am Vor- oder Nachmittag zu arbeiten. In Hausarztpraxen werden neue Modelle der Arbeitszeit und der Praxisöffnungszeiten geschaffen, die den Ärzten und auch den Patienten zugutekommen. Die Konsultationszeiten werden sich auch den geänderten Bedürfnissen der Patienten anpassen müssen. Sie sind heute nicht mehr ohne Weiteres bereit, auf der Arbeit zu fehlen, weil sie zum Arzt müssen. Das Bedürfnis nach Konsultationen in der Früh, also vor $8 \mathrm{Uhr}$, und spät, d.h. nach 17 Uhr, wird wachsen. Darüber kann man jammern oder aber aus der Not eine Tugend machen.

Oft ist die Rede davon, dass sich Hausärzte zu Dienstleistern im Gesundheitsbereich entwickeln müssen. Ist das eine neue Herausforderung?

Nein, denn das sind die Hausärzte schon in der Vergangenheit gewesen - und auch heute. Bei der älteren Generation der Hausärzte erlebe ich eine sehr grosse Bereitschaft, sich um ihre Patienten zu kümmern, sie auch ausserhalb der regulären Sprechstundenzeiten zu sehen oder sogar noch $\mathrm{zu}$ Hausbesuchen $\mathrm{zu}$ 
fahren. Sie stellen oft ihr Privatleben zurück. Ich habe den Eindruck, dass sich Ärzte der mittleren Generation stärker gegenüber den Ansprüchen der Patienten abgrenzen und nach $18 \mathrm{Uhr}$ den Anrufbeantworter einschalten, wenn sie keinen Dienst haben. Der Unterschied zwischen den alten Hausärzten und denen der mittleren Generation ist der, dass die alten Hausärzte einfach rund um die Uhr arbeiten und die anderen sich sagen: «Okay, ich suche mir ein neues Praxismodell, in dem ich Arbeit und Privatleben irgendwie kombinieren kann.»

\section{Durch das Internet hat sich der Informationsstand der Patienten verbessert, und viele kommen bereits mit einer Diagnose und Therapiewünschen. Wie gehen Sie damit um?}

Es ist sehr anspruchsvoll, die Leute zu betreuen, wenn sie sich bereits informiert haben. Dann ist es notwendig und manchmal auch schwierig, sie vor sich selbst zu schützen, weil sie irgendetwas gegoogelt haben, das sie weder einschätzen noch einordnen können. Sie machen sich dann z.B. grundlos Sorgen oder verlangen nach irgendeiner Behandlung, von der sie gehört haben. Ihnen fehlt aber dann oft das Vertrauen, auf den Arzt zu hören, wenn er von einer bestimmten Therapie abrät. Wenn die Patienten überinformiert sind, wird unsere Arbeit als Hausarzt schwieriger.

Ganzheitliche Behandlungsmethoden werden sehr oft im Internet empfohlen. Sorgt das auch für eine grössere Bereitschaft, sie anzubieten?

Ich glaube nicht, dass in der Medizin die Nachfrage das Angebot generiert; es ist wahrscheinlich umgekehrt: Wenn es ein bestimmtes Therapieangebot gibt, nehmen es Patienten auch eher wahr. Wenn in einem Ort z.B. ein Chinazentrum eröffnet wird, entsteht dadurch eine Nachfrage nach Traditioneller Chinesischer Medizin, und die Klientel ist plötzlich vorhanden. Ich glaube, dass es bei den Menschen in der Schweiz ein sehr grosses Bedürfnis nach Massnahmen gibt, die die Gesundheit erhalten. Deshalb werden wahrscheinlich neue Angebote so schnell und gut angenommen.

\section{Springen die zukünftigen Haus- ärzte auf den Zug auf und bieten entsprechende Therapien an?}

Das glaube ich nicht. Ich habe noch nie gehört, dass ein Hausarzt nur wegen der schönen Geschäftsidee beispielsweise Homöopathie anbietet. In der Regel hat man vorbestehend ein besonderes medizinisches Interesse, und daraus entwickelt sich dann eine Spezialität. Viele Hausärzte haben ein medizinisches Steckenpferd, das sie als Spezialität in ihre Praxis integriert haben. Das kann z.B. Komplementärmedizin, Psychotherapie oder manuelle Medizin sein. Man muss vernünftig ausgebildet sein, wenn man so etwas anbieten will. Die meisten Hausärzte betreiben ihr Spezialgebiet mit grosser Seriosität und Ernsthaftigkeit.

Wie sehen Sie die Zusammenarbeit der Hausärzte mit Anbietern von Komplementärmedizin - seien es Chinazentren, Homöopathen oder Phytotherapeuten?

Wenn sie das seriös ausgebildet betreiben, gibt es dort wahrscheinlich in Zukunft wenige Berührungsängste. Es gibt ja Patienten mit Beschwerden, denen wir mit der klassischen Schulmedizin einfach nicht helfen können und denen wir auch nicht gerecht werden. Im Spital sehe ich viele Menschen, die leiden, ohne dass ich in meinem schulmedizinischen Vokabular einen Namen dafür hätte.

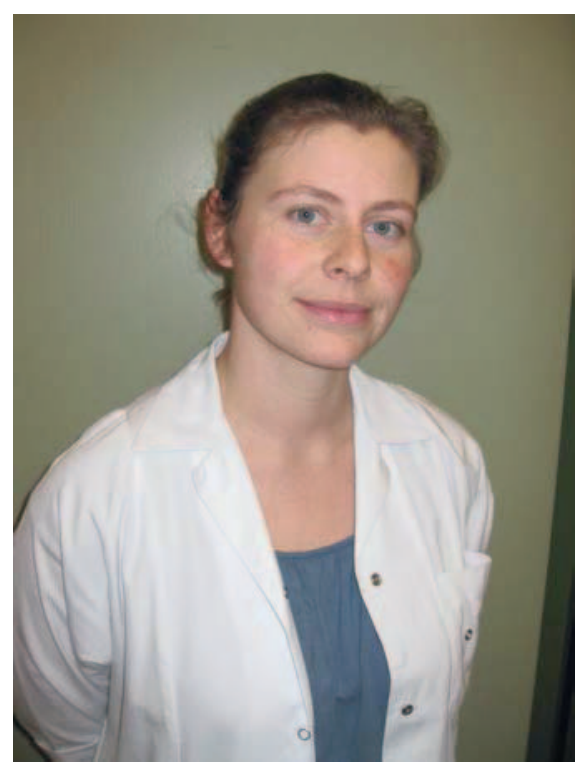

Für sie sehe ich die Möglichkeit, dass sich in der Komplementärmedizin ein Ansatz findet, der ihnen Linderung verschafft.

Dabei denken Sie an psychosomatische Beschwerden, bei denen man mit herkömmlichen Methoden der Schulmedizin schlecht weiterkommt?

Körper und Geist lassen sich kaum trennen. Somit haben die meisten gesundheitlichen Probleme eine psychosomatische Dimension. Es gehört zu den Kernkompetenzen der Hausarztmedizin, das Leiden der Menschen in ihrem komplexen sozialen Umfeld zu erfassen. Die Betreuung von sogenannten psychosomatischen Patienten ist oftmals mühsam und unbeliebt. Diese «schwierigen» Patienten einfach an einen Komplementärmediziner abzuschieben, ist da keine Lösung. Ich denke mehr an konkret umschriebene Fragestellungen an den Komplementärmediziner. Kürzlich habe ich z.B. einen Patienten behandelt, der ein Gefässleiden hat und seit Jahren vergeblich versucht, mit dem Rauchen aufzuhören, wobei er die ganze Palette pharmakologischer Methoden ausprobiert hat. Ich habe ihn gefragt, ob er vielleicht 
Interesse hätte, es mit der Akupunktur $\mathrm{zu}$ versuchen. Daraufhin habe ich ihn an einen Akupunkteur überwiesen, der gut ausgebildet ist und seine Arbeit seriös macht. Leider raucht der Patient immer noch.

\section{Und den alten Streit um Komple- mentär- und Schulmedizin sehen Sie eher pragmatisch?}

Für mich hat es den nie gegeben. Wer therapeutisch tätig sein will, muss entsprechend ausgebildet sein. Der Versuch einer Hobby-Phytotherapeutin, den schweren Heuschnupfen ihrer Freundin mit einem PollenPräparat $\mathrm{zu}$ heilen, hat mir auch schon nächtliche Arbeit und der Freundin einen Ausflug auf die Intensivstation beschert. Egal, mit welcher Form von Medizin man sich beschäftigt, man muss sich der möglichen Wirkungen und Nebenwirkungen seiner Therapie bewusst sein. Erst recht, wenn Therapieleistungen via Krankenkasse von der Allgemeinheit bezahlt werden. Dann sind für mich eine staatlich kontrollierte Ausbildung, eine geregelte Weiterbildung und ein klar umschriebener Leistungskatalog ein Muss. Wenn ein Fach aber seriös betrieben wird, stehe ich zum Motto: Wer heilt, hat recht.

Als Folge des neuen Verfassungsartikels muss Komplementärmedizin an den Universitäten in der Ausbildung der Mediziner angeboten werden. Was halten Sie davon, dass noch mehr Stoff ins Studium kommt?

Ich bin da sehr ambivalent. Die Breite der Medizin, die wir heutzutage haben, ist unglaublich und kaum noch $\mathrm{zu}$ überblicken. In der Ausbildung den Basisstoff zu vermitteln, den man später braucht, ist heute schon schwierig geworden, weil die Zeit an allen Orten fehlt. Vor 30, 40 Jahren hat man sich in der Anatomie ein halbes Jahr lang mit der Schädelbasis beschäftigt - wir hatten im Studium dazu 3 Wochen zur Verfügung. Das Beispiel zeigt das Dilemma, dass schon heute für die Grundlagen nicht mehr genügend Zeit vorhanden ist. Das Feld der Medizin ist so weit, dass es den Rahmen sprengen würde, wenn man künftig verschiedene Bereiche der Komplementärmedizin obligatorisch und auf seriöse Art und Weise in das Studium integrieren würde. Eine gute Alternative wäre, wenn man z.B. die Möglichkeit schaffen könnte, um in Freifächern seinen Interessen nachzugehen. Etwas Ähnliches wird in Bern auf dem Gebiet der Komplementärmedizin bereits durch die Kollegiale Instanz für Komplementärmedizin (KIKOM) angeboten. Ihre Kurse sind eine gute Möglichkeit, sich in das Thema Komplementärmedizin einzuarbeiten.

Welche Rolle spielt für Ihre Weiterbildung die Technik? Haben iPadApplikationen und Online-Tools Kongresse überflüssig gemacht?
Auf keinen Fall, und ich finde, man sollte unbedingt an Kongresse reisen, denn es ist der Austausch mit dem Gegenüber, der einen fachlich und persönlich weiterbringt. Die Diskussionen mit den Kollegen erweitern den Horizont. Den direkten Kontakt wird das Internet nicht ersetzen. Auch auf der Homepage der Jungen Hausärzte versuchen wir immer wieder, Diskussionen im Internet-Forum zu führen. Die schlafen aber meistens rasch ein. Hier ist der persönliche Kontakt nach wie vor einfacher und bringt unter dem Strich mehr.

Der Einfluss der Technik wird eher beim Nachschlagen von Spezialwissen weiter zunehmen. Kein Arzt weiss mehr alles, was er im medizinischen Alltag wissen muss, und da geht es Medizinern nicht anders als Menschen in anderen Berufen. Für Ärzte gibt es eine Reihe von guten Datenbanken wie das Swiss Medical Forum oder UpToDate ${ }^{\circledR}$, wo man $\mathrm{zu}$ vielen Themen gute Artikel findet.

Insgesamt beschreiben Sie Ihre Generation künftiger Hausärzte als sehr verantwortungsvoll und als Gesundheitsdienstleister, die sich für die Patienten engagieren.

Das ist so. Ich denke ohnehin: Auch unsere Generation möchte vor allem einfach gute Medizin für unsere Patienten machen. Und da hat sich wohl im Vergleich zu unseren Vorgängern nichts Grundsätzliches geändert.

Interview: Oliver Klaffke 